More than 50 feet of film was obtained, with above 2000 successive pictures of a region around Copernicus, about 200 by 330 miles in size, the ring-plain being in the middle. Guiding was with a power of 700 and a special eyepiece, which received light from a cube prism just ahead of the film, the diagonal surface of which was lightly silvered to divert about half of the light.

The exposure was too short to show the faint illumination on the low-lying areas outside Copernicus at the exact terminator; but the retreat of the shadow of the 'eastern' ridge down the inner slope of the 'western ' ridge is well depicted. The age of the moon was $9 \cdot 0$ d. on May 18 at 6 h. G.C.T. The guiding was only fair, but the positive made from the film makes a good show when projected. (It will be remembered that Jupiter has already been depicted in motion pictures by W. H. Wright.)

Further development of the apparatus is planned. The low illumination at the terminator offers the chief difficulty, because if the exposure is made long, the whole film is rather short when projected. Technically satisfactory pictures may be helpful to serious investigators of the detailed topography; but the vivid general interest possessed by moving pictures of the moon justifies the effort of securing them.

R. F. ArNotT.

E. G. F. ARNOTT.

Princeton, N.J.,

A. L. Bennett.

June 10

J. Q. Stewart.

\section{Heisenberg's Indetermination Principle and the Quantum.}

THe Heisenberg principle states that $\delta p \delta q \sim h$, where $p$ and $q$ are two canonical conjugates. Energy and time are such conjugates. For the quantum, $E=h \nu$ and we have

$$
\delta(h v) \delta t \sim h \text { or } \delta \nu \delta t \sim 1
$$

where $t$ is the time the quantum has been travelling from its source. If $x$ is the distance the quantum has travelled in time $t$, then $\delta t=\delta x / c$, where $c$ is the velocity of light, so that

$$
\delta \nu \delta x \sim c \text { or } \delta \lambda \delta x \sim \lambda^{2}
$$

since $\delta \nu=c \delta \lambda / \lambda^{2}$, neglecting sign.

From the classical theory (Compton, "X-Rays and Electrons ", p. 55) the amplitude of a radiating oscillator decreases according to $A=A_{0} \epsilon^{-k t}$ due to damping, where $k=\left(4 \pi^{2} / 3\right) \cdot\left(e^{2} / m c \lambda^{2}\right)$ and $e$ is the charge on the electron. The effective length of a wave-train may be taken as the length for $A$ to be reduced to $A_{0} / \epsilon$ and is $c / k$. This length may be taken as the uncertainty $\delta x$ of the position of the quantum, so that

$$
\delta \lambda \sim k \lambda^{2} / c \text { or } \delta \lambda \sim\left(4 \pi^{2} / 3\right) .\left(e^{2} / m c^{2}\right) .
$$

I have previously calculated the width of a spectrum line due to damping by the method of the Fourier integral (Phys. Rev., 19, 64; 1922) and have obtained a width $\Delta \lambda$ between half values of the maximum intensity of

$$
\Delta \lambda=(4 \pi / 3) \cdot\left(e^{2} / m c^{2}\right)
$$

It is interesting to note that $\delta \lambda$ and $\Delta \lambda$ are of the same order, namely, $10^{-12} \mathrm{~cm}$. Does the Heisenberg principle therefore imply that a spectrum line given out by, say, a mercury atom, has a width in addition to the width due to the Doppler and pressure effects ? Can a single quantum itself have a frequency width ? When mercury resonance radiation is absorbed in mercury vapour, it seems necessary to assume a width for a single quantum if any absorption at all is to occur.

$$
\text { Washington University, }
$$

St. Louis, Mo., U.S.A., May 24.

$$
\text { No. 3115, VoL. 124] }
$$

G. E. M. JAUNCEY.
Vibrational Quantum Analysis of Red Gyanogen Bands.

A STUDY of the red cyanogen bands shows that the band at $\nu 14430$ is not the true $O-O$ band of the system. Six new bands on the higher $\lambda$ side have been photographed on neocyanine plates, from the

\begin{tabular}{|c|c|c|c|c|c|}
\hline$n^{\prime} / n^{\prime \prime}$. & 0. & 1. & 2. & 3. & 4. \\
\hline $\begin{array}{l}0 \\
1 \\
2 \\
3 \\
4 \\
5\end{array}$ & $\begin{array}{l}10937(a) \\
12697(a) \\
14432 \\
16143\end{array}$ & $\begin{array}{l}10654(b) \\
12393(a) \\
14099 \\
15788\end{array}$ & $\begin{array}{l}12086(a) \\
13769 \\
15430\end{array}$ & $\begin{array}{l}11782(a) \\
13439\end{array}$ & $11480(a)$ \\
\hline
\end{tabular}
spectrum of cyanogen as developed in active nitrogen (R.K.A.) and of the high current density arc (J.W.R.). These can be fitted in a simple manner in the existing $n^{\prime} / n^{\prime \prime}$ table for the red cyanogen bands, by extending the $n^{\prime}$ progressions as under :

Numbers denote $\nu$ of the most refrangible head. (a) denotes new bands found in active nitrogen and in arc.
(b) found in arc only.

This arrangement does not invalidate the existing analysis completely, and appears to be satisfactory. It is not certain that the band at $\nu 10937$ is the $O-O$ band, which may be higher up in the infra-red, beyond the range of sensitivity of the photographic plates now available. $\omega_{0}^{\prime \prime}$ and $\omega_{0}^{\prime \prime} x^{\prime \prime}$ retain their old values; $\omega_{0}^{\prime}$ is uncertain, but $\omega_{0}^{\prime} x^{\prime}$ retains the old value. The vibrational equation deduced from the present experiments, assuming $\nu 10937$ to be the $O-O$ band, is :

$\nu_{\text {(head) }}=10937+\left(1782 n^{\prime}-13 \cdot 5 n^{\prime 2}\right)-\left(2055 n^{\prime \prime}-13 \cdot 3 n^{\prime \prime 2}\right)$.

Wheatstone Laboratory,

R. K. Asundi.

King's College,

London, W.C.2

Research Laboratories of the

J. W. RYDE.

\section{General Electric Co., Ltd.,} Wembley.

\section{The Raman Effect in Carbon Disulphide.}

IN view of the interesting observations of Rasetti with carbon dioxide (Nature, Feb. 9 . 1929), the Raman spectrum of which was found to contain no lines corresponding to any of the observed infra-red absorption bands, the results obtained in the analogous compound carbon disulphide may be noteworthy. The scattered spectrum of this liquid gives two prominent frequency shifts, 655 and 800 , corresponding to $15.27 \mu$ and $12.50 \mu$ respectively. The infra-red absorption of the liquid was studied by Coblentz in the region $2 \mu$ to $15 \mu$, and he gives the following values for the absorption bands $-3 \cdot 2 \mu, 4 \cdot 6 \mu, 6.8 \mu$, $11 \cdot 65 \mu$, and $13.4 \mu$. All these bands, except the first, are very strong, the one at $6.8 \mu$ being the most prominent. It is significant that none of these bands are represented in the scattered spectrum.

The Raman line corresponding to the frequency shift 655 , excited by 4358 , is very strong in the spectrum and has an anti-Stokes' line the intensity of which is less than 1/50 of it. The value calculated for the intensity ratio between the negative and the positive lines according to the relation $e^{-h v / k T}$ comes out to be 1/20. Whether this discrepancy in the relative intensity has any connexion with the absence of the corresponding infra-red absorption remains to be determined.

210 Bowbazar Street,

A. GavesaN.

Calcutta, May 23.
S. Venkateswaran. 